\title{
CALIZAS ESTROMATOLÍTICAS Y OOLÍTICAS Y DEFINICIÓN DE LA FORMACIÓN ARROYO DE LA PEDRERA (?VENDIANO, URUGUAY)
}

\section{JUAN MONTAÑA* \& PETER SPRECHMANN**}

\begin{abstract}
STROMATOLITHIC AND OOLITHIC, CARBONATIC AND DEFINITION OF THE ARROYO DE LA PEDRERA FORMATION (VENDÍAN?, URUGUAY). The Arroyo de la Pedrera Formation is defined as a new Vendian lithostratigraphic unit of Uruguay. The depositional systems are raade up of: (a) a shallow siliciclastic shelf formed by orthoquarzites and mudstones, grouped in the Cerros San Francisco Member; and (b) peritidal carbonates, which define the Cerro Victoria Member, whose depositional systems extends from the shallow subtidal to the supratidal. They are recognized by oolites, stromatolitic associations and scarce trace fossils. An intermitent subaerial exposition is recognized by tepee-structures, balite pseudomorphs and other features. The sediments are arranged in a shallowing - upward sequence, interrupted by tempestites, developed on a stable shelf, located on the margin of the Rio de la Plata Craton. The age of the formation is inferred by stratigraphic relationships and trace fossils. Deposition took place in a warm water environment, implying low paleolatitudes.
\end{abstract}

Keywords: Siliciclastic shelf, peritidal carbonates, trace fossils, diagenesis, basin analysis, paleotemperatures, paleolatitudes.

\begin{abstract}
RESUMEN Se define una nueva unidad litoestratigráfica para el Uruguay, denominada Formación Arroyo de la Pedrera, tentativamente asignada al Vendiano. Se identifican los siguientes sistemas depositacipnales: (a) una plataforma siliciclástica somera, formada por ortocuarcitas y pelitas las que se agrupan en el Miembro Cerros San Francisco; y (b) carbonates peritidales que tipifican el Miembro Cerro Victoria. Presentan calcarenitas oolíticas, facies estromatolíticas y existió exposición subaérea intermitente, reconocida a partir de estructuras tepee y pseudomorfos de halita. En su conjunto, los sedimentos se disponen en secuencia progresivamente somera (shallowing - upward sequence), interrumpida por tempestitas, desarrolladas en el margen del Craton del Río de la Plata. La edad se infiere por relaciones tectónicas y por icnofósiles. La depositación tuvo lugar en ambientes de aguas cálidas, lo cual implica paleolatitudes bajas.
\end{abstract}

Palabras clave: Plataforma siliciclástica, carbonates peritidales, icnofósiles, diagenesis, análisis de cuenca, paleotemperaturas, paleolatitudes.

INTRODUCCIÓN Las investigaciones que condujeron a este trabajo forman parte del: (a) Programa de investigación "Evolución del Paleozoico Inferior y Medio del Uruguay" (EPIMU), desarrollado en base a un convenio entre la Cátedra de Geología de la Facultad de Agronomía y el Departamento de Paleontología de la Facultad de Ciencias, y (b) Proyecto "Estratigrafía y Paleoecología de las Secuencias Marinas del Paleozoico y Cenozoico del Uruguay", radicado en el citado Departamento de Paleontología $\mathrm{y}$ financiado por la Comisión Sectorial de Investigación Científica (CSIC) de la Universidad de la República.

ANTECEDENTES Los afloramientos estudiados se ubican al NW de la Localidad de Illescas (Fig. 1). El área ha sido cartografiado dentro de la Unidad "Piedras de Afilar", en los mapas geológicos del Uruguay (Bossi et al 1975; Preciozzi et al. 1985). En la misma región, Bossi \& Campal (1992) han estudiado el magmatismo y la tectónica transcurrente del Uruguay durante el Paleozoico Inferior, asociados a una unidad alóctona tectonoestratigráfica denominada "Terreno de Nico Pérez", a la cual pertenecen las rocas descritas en este trabajo.

FORMACIÓN ARROYO DE LA PEDRERA Definición Conjunto compuesto, desde la base al techo, por cuarcitas, pelitas y calizas oolíticas y estromatolíticas depositadas en forma concordante (Fig. 2 y 3 ).

Ubicación geográfica y tectonismo Unidad litoestratigráfica depositada en discordancia sobre rocas graníticas fuertemente tectonizadas y milonitizadas, con estira- miento predominante de cristales en el sentido N20W. El principal accidente es la falla transcurrente dextral Sarandí del Yí -Piriápolis, larga y estrecha faja estructural de varios miles de metros de ancho, la cual limita al Terreno de Nico Pérez por el oeste (Fig. 1, punto A). Otra importante zona de cizallamiento, paralela a la anterior (Fig. 1, punto B), ha sido datada en $540 \pm 25 \mathrm{Ma}$ (Fig. 1, punto C) (Bossi \& Campal 1992 apud Umpierre \& Halpern 1971, Bossi \& Campal 1993). Esta zona de cizallamiento afecta a los sedimentos de la Formación Arroyo de la Pedrera, demostrando que su depositación fue anterior. La citada zona de cizallamiento reaparece hacia el norte, paralela al oeste del Arroyo San Francisco. Las rocas sedimentarias de la Formación Arroyo de la Pedrera han sido afectadas por esfuerzos tectónicos que las plegaron, formando un enorme sinclinal cuyo eje tiene rumbo N15E. Los buzamientos del citado sinclinal se ilustran en la figura 1.

$\mathrm{El}$ área de afloramientos se encuentra al W, delimitada por el lineamiento Sarandí del Yí - Piriápolis; al N y NE, por fallas de rumbo N35 W que se desarrollan paralelas al Arroyo Illescas; Al S, queda delimitada por el Arroyo Arrayán y la vía férrea. El area cubierta por la formación en la zona referida es de unos $56 \mathrm{~km}$ cuadrados.

Espesor La unidad posee potencia de 350 a $380 \mathrm{~m}$.

Subdivisión litoestratigráfica La Formación Arroyo de la Pedrera se divide en dos miembros, a saber: (a) Miembro Cerros San Francisco y (b) Miembro Cerro Victoria.

MIEMBRO CERROS SAN FRANCISCO (Fig. 1 y 2) Caracterizado por metasedimentos siliciclásticos. Presenta areniscas medias y areniscas finas subordinadas, en

\footnotetext{
* Facultad de Agronomía, Cátedra de Geología, Avenida Garzón 780, Montevideo, Uruguay

** Departamento de Paleontología, Facultad de Ciencias, Tristán Narvaja 1674, 11200 Montevideo, Uruguay
} 


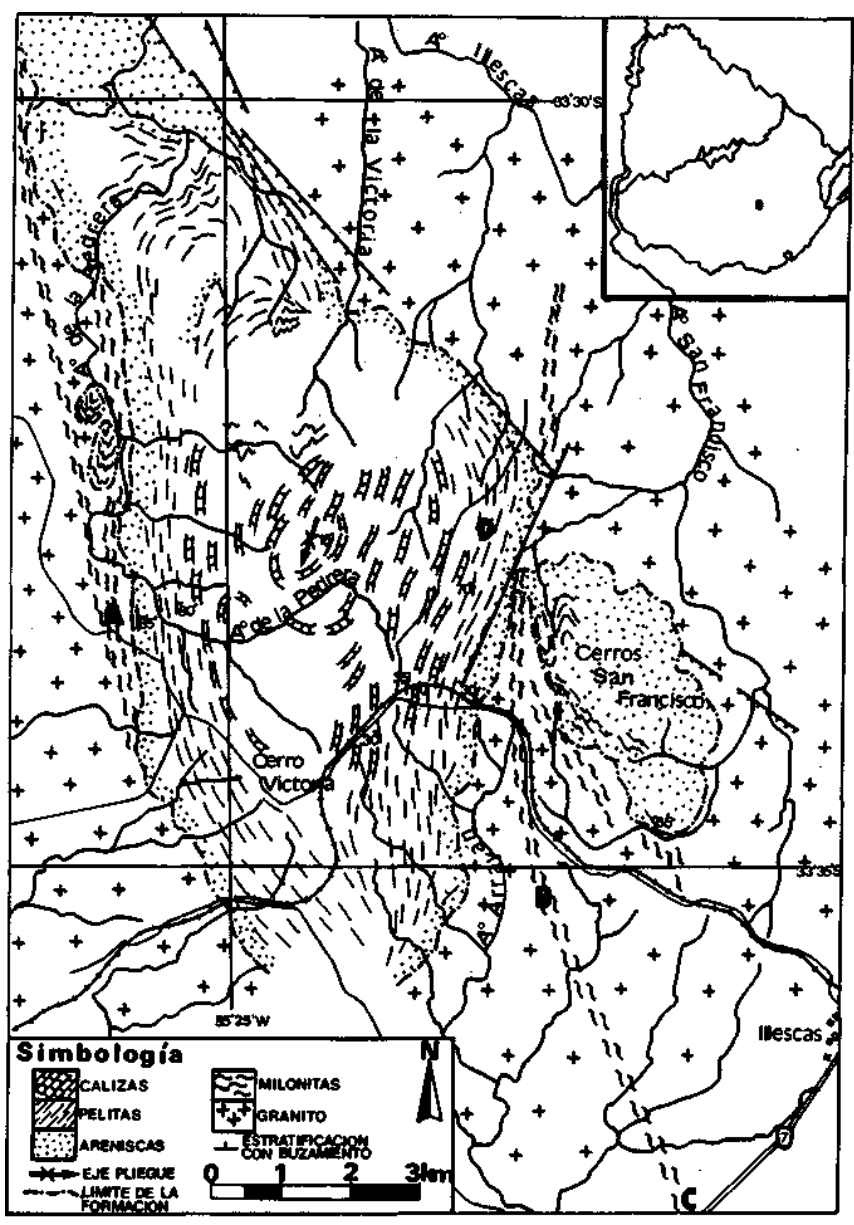

Figura 1 - Mapa geológico de la Formación Arroyo de la Pedrera

Figure 1 - Geological map of the Arroyo de la Pedrera Formation

la base, de colores grises muy claros y blancos (N8 y N9), las que gradualmente pasan a areniscas muy finas y pelitas de colores rojizos tenues (10 R 6/2). Las areniscas básales, clasifícables como ortocuarcitas (véase Addenda). Poseen muy escasa matriz arcillosa. Presentan estratificación decimétrica a métrica, con superficies de estratificación planas, esporádicamente tapizadas por pelitas. Con frecuencia se hallan, en la base de los estratos, intraclastos discoidales o irregulares de pelita. En ellas se identifican artesas, en estructuras tridimensionales, producidas por migración de ondas medias de arena. También se visualizan estratos tangenciales simples, en expresión bidimensional. Algunos de los estratos de areniscas, en el tope, presentan ondulitas asimétricas, denotando paulatina disminución de la energía. Existe pasaje gradual, de 5 a $10 \mathrm{~m}$, a metapelitas, constituyendo estratos bien delimitados, y a areniscas muy finas de colores rojizos tenues (10 R 5/4), bien sorteadas, con moderada matriz, con estratificación fina y gradación normal. En ellas existen ondulitas asimétricas aisladas. En su conjunto, el Miembro Cerros San Francisco representa una secuencia moderadamente granodecreciente, la cual, junto a las estructuras sedimentarias descritas, denota disminución de la energía. El miembro se caracteriza por la presencia de numerosos filones de cuarzo blanco, de considerable longitud y ancho métrico, que recortan por igual al basamento como a los sedimentos. Otras manifestaciones se denotan en las cuarcitas, como ser venillas grises de cuarzo de 5 a $15 \mathrm{~cm}$ de largo y 1 a $2 \mathrm{~cm}$ de ancho, subhorizontales y, a veces, ramificadas. La génesis de las venillas se interpreta como debida a procesos diagenéticos.

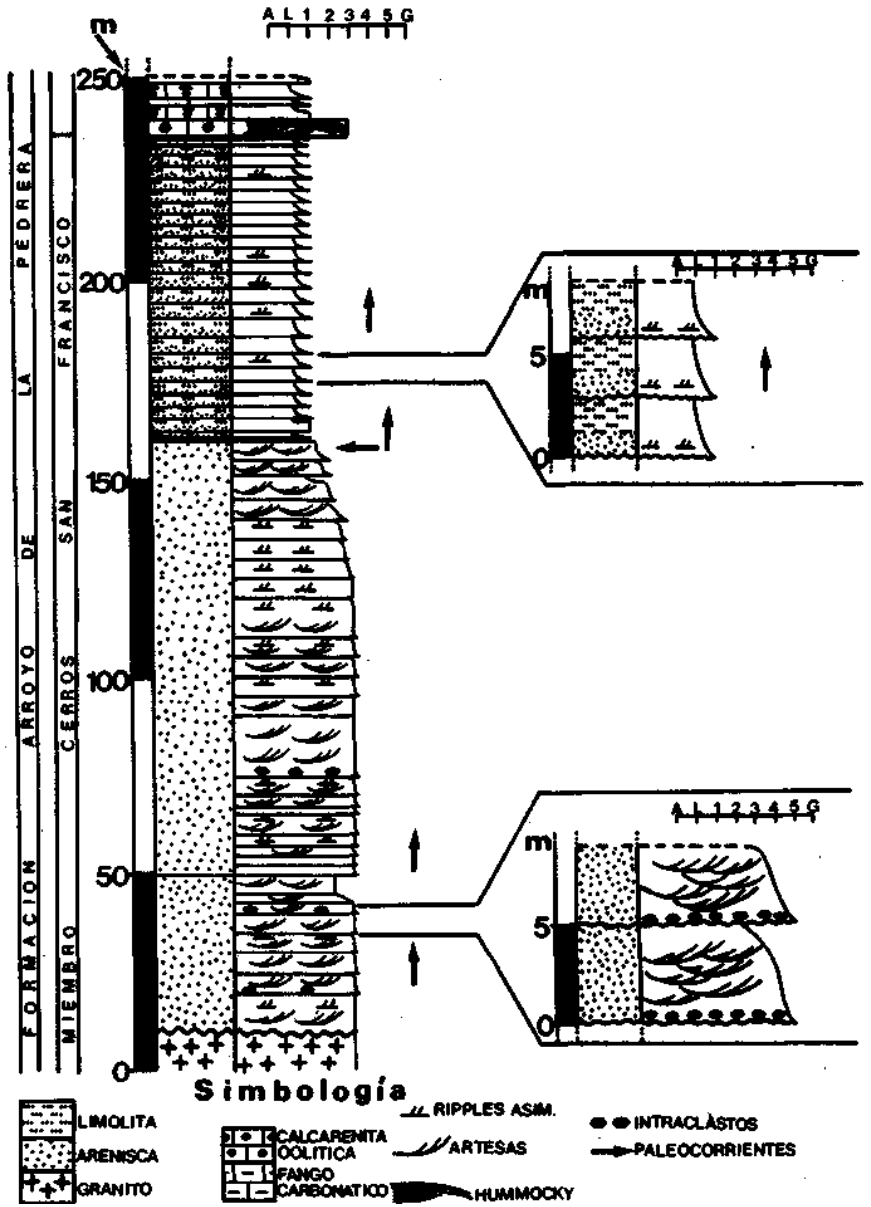

Figura 2 - Columna estratigráfica del Miembro Cerros San Francisco

Figure 2 - Columnar section of the Cerros San Francisco Member

MIEMBRO CERRO VICTORIA (Fig. 3) El inicio de la sedimentación carbonatada caracteriza al Miembro Cerro Victoria. En la base, se encuentra formado por sedimentos formados a partir de calcarenitas oolíticas y barros calcáreos, ambos producidos por la removilización de materiales provenientes de ambientes de mayor energía. Las calcarenitas oolíticas, de color amarillo (10 YR 6/6), debido a la presencia de limonita, conforman una secuencia granocreciente, a partir de oolitos de grano medio, con cemento esparítico, hasta llegar a arenas oolíticas casi puras, de grano grueso, clasto soportadas. Estas últimas presentan incluidas fragmentos de láminas pertenecientes a estromatolitos.

En su conjunto, existe somerización en ambiente de aguas bien iluminadas, agitadas, con energías crecientes. Las calcarenitas oolíticas se asocian a niveles con estratificación hummocky, lo que evidencia la existencia de tempestitas, que potencian la removilización de los oolitos. En los escasos estratos pelíticos intercalados hacia la base del Miembro Cerro Victoria, aparecen pseudomorfos de cristales de halita, evidenciando ocasionales condiciones hiperhalinas supertidales (Fig. 3). Siguen unos $50 \mathrm{~m}$ en los que alternan pelitas calcáreas y barros cada vez más carbonáticos. Dominan colores amarillos y rojizos (10 YR 6/6, 10 R 6/2).

El Miembro Cerro Victoria culmina con más de $50 \mathrm{~m}$ de crecimientos de calizas estromatolíticas, formadores de roca, secundariamente silicificadas. Presentan coloración general gris (N 7). Su laminación gris se intercala con láminas de coloración rosado-naranja-claro (5Y R 8/4), evidenciando respectivamente alternancia de períodos 


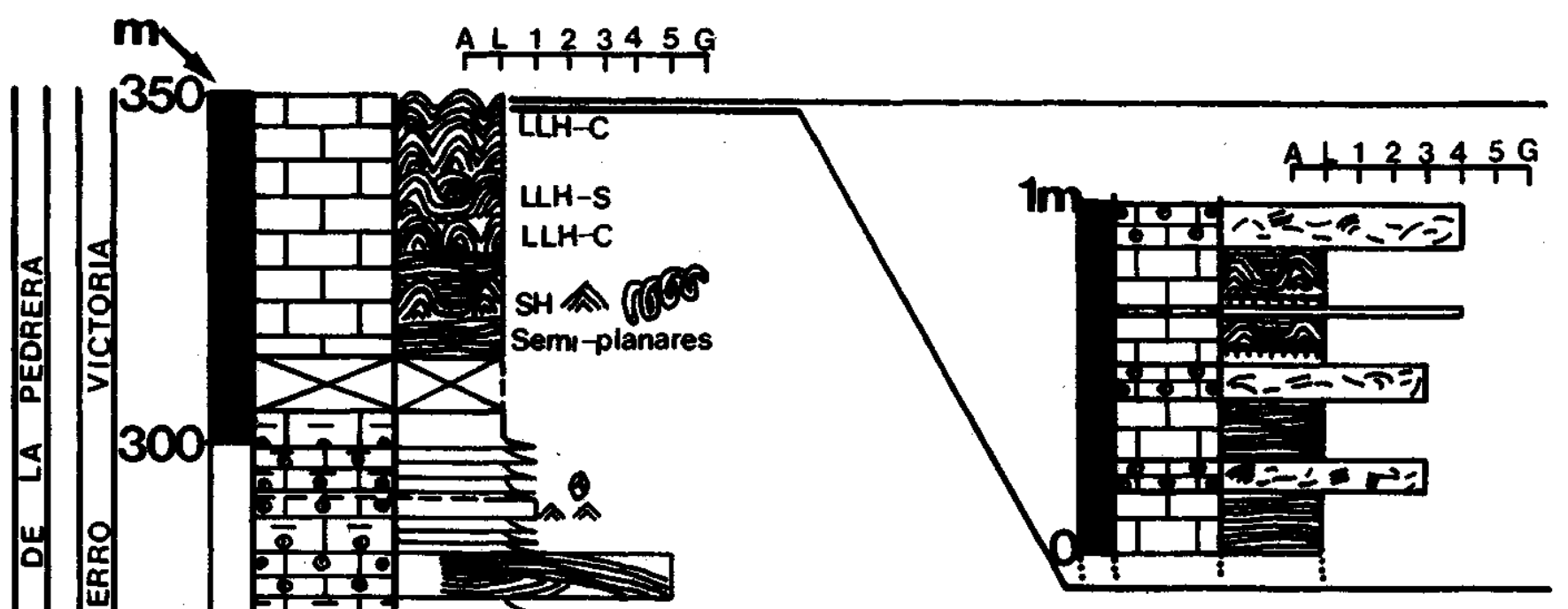

Simbología
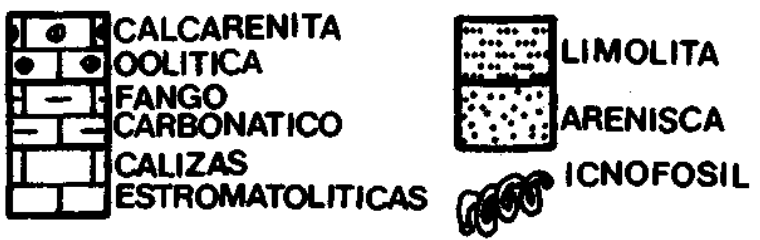

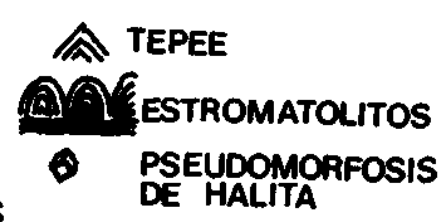

Figura 3 - Columna estratigráfica del Miembro Cerro Victoria Figure 3 - Columnar section of the Cerro Victoria Member

breves, probablemente nictimerales, de exposición aérea. Lateralmente, aparecen calcarenitas muy finas o limos calcáreos, con ondulitas simétricas y levemente asimétricas de cresta roma. Se destaca que los $20 \mathrm{~m}$ superiores se hallan, en su totalidad, secundariamente silicificados. Como consecuencia, es posible hallar en la superficie del área aledaña al punto D (Fig. 1), gran cantidad de fragmentos silicificados de estromatolitos LLH-C,LLH-S y calcarenitas eolíticas gruesas (véase el ítem siguiente). Estas últimas se encuentran intercaladas, con mayor frecuencia, con estromatolitos LLH-S, hallados en posición de crecimiento o fragmentados. Las calcarenitas eolíticas intercaladas referidas pueden alcanzar espesores centimétricos.

Paleontología del Miembro Cerro Victoria Se identifica, por primera vez en Uruguay, lo que constituyen sus fósiles más antiguos. Trátase de crecimientos estromatolíticos. Siguiendo la clasificación de Logan et al (1964), se reconocen:

a. Laminitas cryptalgales, también denominados estromatolitos planares, con estructuras fenestrales.

b. Hemiesferoides unidos lateralmente (LLH-C).

c. Hemiesferoides espaciados unidos lateralmente del tipo LLH-S.

d. Estromatolitos planares sobre los que crecen hemiesferoides con apilamiento vertical SH. e. Existe transición vertical de combinaciones de crecimientos, por ejemplo del tipo a al b y del tipo b al c, constituyendo estromatolitos complejos. Hay a su vez pasajes laterales entre estas formas, como consecuencia de cambios leves del nivel marino,probablemente debidos a cambios estacionales. Como ya se indicó, los tipos descritos caracterizan a más de $50 \mathrm{~m}$ de espesor, con los que culmina el Miembro Cerro Victoria.

f. En las microfacies de calcarenitas, las oolitas son predominantemente esféricas o subesféricas, presentándose también complejas (3 a $5 \%$ ) y cerebroides (1\%). Los núcleos son carbonáticos esparíticos, así como el cemento. 
En el miembro también se encuentran icnofósiles diversos, a saber: a. tubos simples; b. icnofósiles con bifurcaciones engrosadas; c. formas meandriformes; y $\mathbf{d}$. trazas con probable ordenamiento pseudohelicoidal. Todos se encuentran preservados como momias esparíticas, de color rojo claro (5 R 6/6), lo cual dificulta las determinaciones taxonómicas, en particular de las formas c y d. En aquellos casos en los que los icnofósiles se encuentren asociados a estromatolitos planares, debe evitarse la confusión con estructuras fenestrales. Dadas las razones expuestas, sólo es posible identificar las formas más sencillas, que pertenecen a los icnogéneros Gordia, Planolites y Sellaulichnus (cf. Häntzschel 1975, Fedonkin 1981, Crimes 1987).

Ambientes de sedimentación de la Formación Arroyo de la Pedrera 1. El Miembro Basal Cerros San Francisco representa la sedimentación siliciclástica plataformal ingresiva, en una cuenca que se colmata paulatinamente, lo que queda evidenciado por la disminución de la energía, que se manifiesta por el decrecimiento, hacia el tope, de la granulometría y del espesor de los estratos, así como por la distribución de las estructuras sedimentarias (Fig. 2). Estas muestran, en la base, areniscas no fosilíferas, organizadas en artesas, con capas frontales tangenciales simples, muy extendidas, de bajo ángulo, lo que implica depositación subacuática. Además, presentan un patrón unidireccional constante por lo que se infiere que fueron formadas por comentes paralelas a la costa, con dirección norte. En la parte más alta del miembro, las ondulitas asimétricas representan comentes de menor intensidad; están dirigidas hacia el norte así como al oeste. Estas últimas serían originadas por el oleaje. En términos de la estratigrafía genética, el miembro representa a un sistema depositacional de plataforma siliciclástica.

2. Existe pasaje gradual hacia el miembro superior, Cerro Victoria, caracterizado por depositación carbonatada en la cual, prácticamente, están ausentes los siliciclásticos (Fig. 3). El miembro representa una somerización creciente y la exposición a ambientes subaéreos, sea por leves cambios del nivel marino, a escala estacional o anual, y/o a la paulatina colmatación de la cuenca. A su vez, ambos fenómenos podrían estar asociados en el tope de la secuencia, a una caída del nivel marino de amplitud mayor, con la progradación subsiguiente de las facies carbonatadas. La preservación de las facies estromatolíticas fue, también, facilitada por la cementación temprano - diagenética que las caracteriza, así como a fenómenos vadosos (Tucker \& Wright 1990).

En el Miembro Cerro Victoria se encuentran, sucesivamente, los siguientes sistemas depositacionales: a. Subtidal muy somero, de aguas de alta energía, bien iluminado, situado por encima del nivel de base del oleaje del buen tiempo, ocasionalmente afectado por tormentas. Dominan en la parte inferior, los estratos de barros calcáreos, interdigitados con las calcarenitas eolíticas, las que predominan en la sección subtidal superior. En ambientes actuales, la producción de oolitas se produce en aguas agitadas, y se ve facilitada por temperaturas cálidas de 20 a $30^{\circ} \mathrm{C}$, lo cual determina la sobresaturación de Ca por escape del $\mathrm{CO}_{2}$ a la atmósfera. Mayores informaciones sobre los procesos de calcificación en algas y cianobacterias son proporcionadas por Golubic (1991), Pentecost (1991) y Riding (1991).

Naturalmente, no resulta posible aplicar el principio del actualismo en tiempos tan remotos, dada la muy probable presencia de anactualismos (Awramik 1991). b. Intertidal, diagnosticado por la presencia de estromatolitos LLH y SH, así como por los pseudomorfos de cristales de halita, que atestiguan desecación permanente y asociación con ambientes hiperhalinos. También existen estructuras sedimentarias que confirman la existencia de este ambiente de depositación, como ser de ondulitas simétricas y otras levemente asimétricas con cresta roma las que, respectivamente, indican corrientes de marea bidireccionales y playas de baja energía, muy restringidas.

c. Supratidal, o límite supratidal - intertidal, representado por los estromatolitos LLH-C y LLH-S.

Las estructuras estromatolíticas intertidales y supratidales constituyen biostromas.

La existencia de los previamente citados niveles centimétricos de calcarenitas oolíticas intercaladas con los estromatolitos, fundamentalmente del tipo LLH-S, indica que las oolitas quedaron atrapadas en oquedades de las matas estromatolíticas, como consecuencia de episodios extraordinarios, representados por tormentas, que rebasaron el nivel de la barra constituida por el biostroma.

La presencia de la estructura poligonal tepee (véase diagénesis) y coloración rojo pálida (5 R 6/2) de la laminación estromatolítica debida a la oxidación, demuestran la existencia de fenómenos de desecación propia de ambientes peritidales, es decir, intertidales y supratidales.

Diagénesis de los carbonates del Miembro Cerro Victoria La laminación que caracteriza a los estromatolitos es remarcada por la dolomitización, en función de meteorización diferencial, resistiendo más las laminitas que poseen enriquecimiento en $\mathrm{Mg}$. Si se admite el modelo de Poiré (1987) para la dolomitización temprano diagenética de la materia orgánica, las laminitas corresponderían al ordenamiento espacial de esta última, en tanto que los espacios interlaminares poseían una composición de calcita con magnesio. Es así que se presenta alternancia de láminas de calcita con magnesio y dolomita en los estromatolitos, y calcita con magnesio en los espacios intercolumnares y calizas detríticas. Los estromatolitos presentan venillas de calcita, producto de su disolución y ulterior recristalización. En las márgenes de las plataformas someras actuales, como en los bancos de las Bahamas, el $\mathrm{CO}_{2}$ disuelto en el agua marina es movilizado por el aumento de temperatura y la turbulencia por lo que, frecuentemente, se produce activa precipitación inorgánica. Bajíos o lóbulos de arenas oolíticas sumergidas comunmente cementan, formando hardgrounds. Costras de apariencia similar, que poseen aragonita fibrosa y cementos calcíficos con magnesio peloidales se presentan actualmente en las lagunas hiperhalinas del SE del Golfo Pérsico (Scoffin 1987). En ellas existe un alto flujo de agua, un sustrato estable y una tasa de sedimentación reducida. Estos hardgrounds, localmente, desarrollan superficie de estratificación poligonal, debido a su fragmentación en grietas o hendiduras. Constituyen anticlinales y luego fracturamientos y corrimientos, debido a la expansión de la costra inducida por la fuerza de cristalización durante la litificación, o a la contracción térmica (Ferguson et al. apud Tucker \& Wright 1990). El desarrollo secuencial de capas sobrecorridas de hardgrounds producen apilamiento de láminas cóncavas, las que desarrollan perfil típico en tiendas de campaña, por lo que se las denomina estructuras tepee. En el Vendiano pudo haber intervenido un aumento de la temperatura y/o la sobresaturación de las aguas con $\mathrm{CO}_{2}$. En el Miembro Cerro Victoria se identifican hardgrounds asociados a las calcarenitas oolíticas que se reconocen por: a. constituir superficies de abrasión lisas a partir de los oolitos, que cortan en microdiscordancia a estructuras sedimentarias asociadas; b. formar costras que sirvieron como sustrato duro para ulterior crecimiento de los estromatolitos; y c. asociarse a las estructuras tepee descritas, en lo principal, vinculadas a los estromatolitos. En estadios diagenéticos tardíos se produjo la silicificación secundaria mencionada de los $20 \mathrm{~m}$ superiores del Miembro Cerro Victoria.

EDAD A la Formación Arroyo de la Pedrera se le atribuye, tentativamente, edad Vendiana. Su depositación es 
anterior a los $540 \pm 25 \mathrm{Ma}$, por las razones expuestas en el capítulo de ubicación geográfica y tectonismo, aunque debe acotarse de que el dato se basa en una sola datación, y no se puede descartar de que puede tratarse de la edad de una de las reactivaciones de la falla y no la de su origen. En el Miembro Cerro Victoria se identifican los icnogéneros Gordia, Planolites y Sellaulichnus, cuyo rango se inicia en el Vendiano y prosigue en el Fanerozoico, lo cual determina que la depositación es Vendiana o ulterior.

En general, las asociaciones de icnofósiles relacionadas a estromatolitos del Vendiano no resultan ser diagnósticas de edad, lo cual se confirma en este caso. Sin embargo, si fuera posible confirmar la existencia de icnofósiles meandriformes y/o pseudohelicoidales, éstos indicarían edad Tommotiana o más moderna para el Miembro (Fedonkin 1985, Crimes, 1987). La edad podría precisarse con el hallazgo de fósiles indicativos, como ser los que caracterizan a la fauna de Ediacara, o por estudios paleomagnéticos.

Por el momento, resulta imposible aproximar esta datación por medio de hipótesis paleoclimáticas o cinemáticas, dadas las incertidumbres existentes acerca de la ubicación del Cratón del Río de la Plata durante el Neoproterozoico y el Cámbrico Inferior.

CORRELACIONES El Miembro Cerro Victoria probablemente se correlaciona en el Uruguay con las calizas de Polanco, ubicadas $50 \mathrm{~km}$ al SE, y las de la Cuchilla Guazunarnbí, situadas al W en el Departamento de Cerro Largo, que representan áreas de sistema depositacional de plataforma carbonatada normal marina, dentro de la zona fotica, así como con otras facies de metacarbonatos presentes en el Grupo Lavalleja. En su conjunto, representan la más importante depositación carbonatada de la historia geológica del Uruguay. Incluso, la sucesión estratigráfíca constituida por areniscas en la base, pelitas en la parte media y carbonatos recristalizados en el tope que caracteriza, en su conjunto, a la Formación Arroyo de la Pedrera, es similar a las halladas en Polanco y la Cuchilla Guazunambí.

Hasta el presente, la región correspondiente a la Formación Arroyo de la Pedrera ha sido cartografiada como formando parte de la Formación Piedras de Afilar (Bossi et al. 1975,Preciozzi et al. 1985). Las descripciones existentes permiten afirmar de que no existen en la Formación Piedras de Afilar las litofacies de calizas estromatolíticas y oolíticas que tipifican al Miembro Cerro Victoria (véase Jones 1956, Bossi 1966, Coronel et al. 1982, Bossi \& Navarro 1988, Cingolani et al. 1990).

En Piedras de Afilar solo existen escasos lentes de calizas.

El "banco de areniscas y cuarcitas" sensu Coronel et al (1982) difiere del Miembro Cerros San Francisco al igual que las estructuras sedimentarias dominantes. Las "limolitas" poseen tonalidades muy oscuras, hasta gris verdosas, lo que implica incluso condiciones reductoras a disaeróbicas. Las pelitas del Miembro San Francisco indican ambientes oxidantes. Por otra parte, Coronel et al (1982) señalan que, en la Formación Piedras de Afilar, se han reunido un conjunto de afloramientos entre los que aún no están establecidas las relaciones estratigráficas. Es de señalar que los principales estudios se limitan al área tipo, que se ubica en los alrededores de la Estación Piedras de Afilar. Tampoco existen datos que permitan sostener razonable equivalencia de edad entre ambas formaciones. La mera presencia de ambientes de plataforma y peritidales no habilita una correlación a priori. Estos criterios justifican la creación de la Formación Arroyo de la Pedrera como unidad independiente, máxime si se analizan los rangos de tiempo involucrados, que pueden llegar a abarcar desde parte del Vendiano al Ordovícico.

ESTRATOTIPOS Se designa como estratotipo de la Formación Arroyo de la Pedrera a las nacientes del curso de agua homónimo. Como paraestratotipo al tercio inferior del Arroyo de la Pedrera hasta unos $1.000 \mathrm{~m}$ de su desembocadura en el Arroyo Illescas. En el estratotipo y paraestratotipo se incluyen a los miembros, con excepción del paraestratotipo del Miembro Cerro Victoria, el que se sitúa en la cañada que vuelca sus aguas hacia el E, que corta el camino a $4 \mathrm{~km}$ de la parada del km 195 de la línea férrea (Fig. 1, punto D). Como área o región tipo se propone a la mapeada en la figura 1.

\section{ANÁLISIS DE CUENCA Y PALEOGEOGRAFÍA LaS}

ortocuarcitas del Miembro Cerros San Francisco se caracterizan por su gran madurez textural, conteniendo $90 \%$ de cuarzo (Gaucher \& Schipilov, Addenda). Esto coincide con la situación presente a escala global. Debe destacarse que las areniscas cratónicas del Precámbrico y Cámbrico se encuentran entre las más maduras del planeta. Ellas son notables por su redondez y el sorteo de los granos, y por poseer hasta $99 \%$ de cuarzo, presentando únicamente trazas de otros minerales estables.

Los minerales presentes atestiguan áreas de aporte metamórficas y graníticas. Sin embargo, los granos probablemente posean larga y compleja historia sedimentaria previa a su depositación definitiva. La redondez manifiesta presente en los granos de las areniscas muy finas, finas y medias demuestran que fueron - al menos las de granu-lometrías más finas - transportadas primariamente por el viento. Este agente es 100 veces más efectivo que el agua para redondear a los citados granos de cuarzo, viéndose favorecida su acción por la ausencia de vegetación en los continentes. Al componente aventado, se agregó la abrasión de la fracción arena generada por la acción de comentes marinas. Las areniscas del Miembro Cerros San Francisco se tipifican por la presencia de artesas decamétricas, con patrón de corrientes muy constante hacia el Norte, formadas a partir de corrientes paralelas a la costa. Están subrepresentadas las estructuras sedimentarias indicativas de comentes de marea y/o acción del oleaje.

Resumiendo, se postula que las arenas del Miembro Cerros San Francisco poseen compleja historia depositacional,con componentes de arenas continentales, las que fueron aventadas a cuencas marinas o transportadas por ríos y retrabajadas, junto con otras primariamente depositadas en el fondo marino por el megaciclo transgresivo descrito en la figura 2. Las pelitas se formaron a partir de la meteorización de los granitos y metamorfitos.

Paulatinamente, decreció la depositación de materiales terrígenos. Cesó de funcionar la "gran máquina transportadora de arena". En el margen del Cratón del Río de la Plata comenzó a funcionar la "máquina de carbonatos", formándose depósitos muy someros de este sedimento. Es muy probable que se haya instalado extensa plataforma carbonatada. Hacia la costa la circulación se vio restringida, apareciendo las litofacies del Miembro Cerro Victoria.

Puede concluirse que existen evidencias de paleotemperaturas cálidas, a partir de elevada tasa de evaporación. En las ortocuarcitas del Miembro Cerros San Francisco, se patentiza alta tasa de evaporación dada la presencia de $6 \%$ de cemento temprano-diagenético de yeso véase Gaucher \& Schipilov (Addenda).

Como se indicara, en la base del Miembro Cerro Victoria sobreyacente, dominan calcarenitas oolíticas, intercaladas con estratos pelíticos en los que aparecen pseudomorfos de halita, que se interpretan como evidencia de condiciones hiperhalinas supratidales, y que refuerzan la interpretación señalada. Todo ello implica, dada la constancia del fenómeno, paleotemperaturas cálidas, o menos probablemente, templado-cálidas. Por lo tanto, se postula para el Cratón del Río de la Plata paleolatitud baja, siendo menos plausible la hipótesis de cuenca restringida. 
Ambas conclusiones poseen implicancias paleogeográficas. En particular la primera, más verosímil en relación a la paleolatitud baja, se ve reforzada si se confirmara la isocronía de importante plataforma carbonatada, representada por las calizas de Polanco, en el margen del Gratén del Río de la Plata.

CONCLUSIONES Se crea la Formación Arroyo de la Pedrera, tentativamente atribuida al Vendiano, que ha sido cartografiada en dos regiones: (1) NW de Illescas y (2) en las inmediaciones del Arroyo del Soldado. Esta unidad litoestratigráfica se subdivide en:

a. Miembro Cerros San Francisco, formado a partir de areniscas clasificables como ortocuarcitas y pelitas, representando secuencia moderadamente estrato y granodecreciente de sistema depositacional de plataforma siliciclástica, y

b. Miembro Cerro Victoria, tipificado por sedimentación carbonatada, donde se destacan calcarenitas eolíticas, asociadas a eventos tempestíticos, y crecimientos estromatolíticos de ambientes propios de sistemas depositacionales subtidales, intertidales y supratidales.

También se identifica una icnofacies de baja diversidad y manifestaciones diagenéticas, como ser hardgrounds, estructuras tepee etc. El Miembro Cerro Victoria probablemente se correlaciona con las calizas de Polanco, que representan las facies de sistema depositacional de plataforma carbonatada normal marina.

En su conjunto, los sedimentos que constituyen la Formación Arroyo de la Pedrera se disponen en secuencia progresivamente somera (shallowing upward sequence), ubicada en el margen del Cratón del Río de la Plata.

Se presentan criterios de diagnóstico diferencial con la Formación Piedras de Afilar.

Asimismo, se efectúa análisis de cuenca que revela compleja historia depositacional. Existen evidencias de tasa de evaporación elevada, lo que implica temperaturas cálidas o, menos probablemente, templado-cálidas. Por lo tanto, se postula para el Cratón del Río de la Plata latitud baja, siendo menos probable la hipótesis de cuenca restringida.

Agradecimientos Al Prof. Dr. Jorge Bossi, el reconocimiento de los autores por importantes indicaciones vinculadas con la distribución de rocas calcáreas en el Uruguay. Importantes sugerencias fueron aportadas por el Prof. Dr. P.C. Soares (Curitiba). Por la eficiente colaboración brindada así como por la realización de los dibujos, se agradece al geólogo. Claudio Gaucher.

\section{REFERENCIAS BIBLIOGRÁFICAS}

AWRAMIK, S.M. 1991. Archean and paleozoic stromatolites. In: RIDING, R. ed. Calcareous Algae and Stromatolites. Berlín, Springer-Verlag. p. 289-304.

BOSSl, J. 1966. Geología del Uruguay. Montevideo, Universidad de la República. $469 \mathrm{p}$.

BOSSI, J. \& CAMPAL, N. 1992. Magmatismo y tectónica transcurrente durante el Paleozoico Inferior en Uruguay. In: GUTIERREZMARCO, J.G.; SAAVEDRA, J.: RÁBANO, I. eds. Paleozoico Inferior de Ibero-América. Extremadura, Universidad de Extremadura, v. 17 , p. $343-356$.

BOSSI, J \& CAMPAL, N. 1993. El Cinturón Cuchilla Dionisio: evento Brasiliano Uruguayo. In: SIMP. INTERN. NEOPROTEROZOICO CÁMBRICO DE LA CUENCA DEL PLATA, 1. Las Palomas Minas, 1993. Guía Exc... Montevideo, p. 41-56.

BOSSI, J. \& NAVARRO, R. 1988. Geologia del Uruguay. Montevideo, Universidad de, la República. $970 \mathrm{p}$.

BOSSI, J.; FERRÁNDOLA.; FERNANDEZ, A.N.; ELIZALDE, G.; MORALES, N. LEDESMA, JJ - CARABALLO E; MEDINAA, E; FORD, L; MONTANA, J.R. 1975 eds. Carta Geológica del Uruguay. Esc. 1:1.000.000. Montevideo

CINGOLANI, C; SPOTURNO, J.; BONHOMME, M. 1990. Resultados mineralógicos y geocronológicos preliminares sobre las unidades Piedras de Afilar, Lavalleja y Barriga Negra, RO del Uruguay. In: CONGR. URUG. GEOL., 1. Montevideo, 1990. Anales... Montevideo, SGU. v. 1, p. 11-15.

CORONEL, N.; OYHANTCABAL, P.; SPOTURNO, S. 1982. Consideraciones estructurales de la Formación Piedras de Afilar en su área tipo. Canelones, Uruguay. In: CONGR. LATINOAMER. GEOL., 5. Buenos Aires, 1982. Actas... Buenos Aires, Sociedad Uruguaya de Geología, v. 5, p. 40-60.

CRIMES, P.T. 1987. Trace fossils and correlation of late Precambrian and early Cambrian strata. Geol. Mag., 124(2):97-119.

FEDONKIN, M.A. 1985. Vendskaja sistema 1, istoriko - geologiceskoe i paleontologiceskoe obosnovanie Palleontologija. Engl. versión: Paleoichnology of Vendían Metazoa. In: SOKOLOV, B.S. \& IWANOVSKI, A.B. ed. The Vendian System. Berlín, Springer-Verlag. v. 1 , p. 132-137.

FEDONKIN, M.A. 1991. White Sea biota of Vendian (Precambrian non ske letal fauna of the Russian Platform North). Moscow, Academy of Science ofthe USSR. p. 1-99. (Transact. 342)
GOLUBIC, S. 1991. Modern stromatolites: a review. In: RIDING, R. ed Calcareous Algae and Stromatolites. Berlin, Springer-Verlag. p. 541-561.

HÄNTZSCHEL, W. 1975 Trace fossils and Problemática. 2 ed. Treatise on Invertebrate Paleontology, part W, Miscellanea, suppl. 1. Boulder, Colorado, Geol. Soc. Am. - University of Kansas. $280 \mathrm{p}$.

JONES, G. 1956. Memoria explicativa y mapa geológico de la región oriental del Departamento de Canelones. Bol. IGU, 34:1 -193.

LOGAN, B.W.; REZAK, R.; GINSBURG, R.N. 1964. Classification and environmental significance of algal stromatolites. J. Geol., 72:62-83.

PENTECOST, A. 1991. Calcification processes in Algae and Cyanobacteria. In: RIDING, R. ed. Calcareous Algae and Stromatolites. Berlin, Springer-Verlag. p. 3-20

POIRE, D.G. 1987. Dolomitización estromatolítica/enterramiento tardío en el Precámbrico de Olavarría, Provincia de Buenos Aires, Argentina. In: CONGR. GEOL. ARGENT., 10. San Miguel de Tucumán, 1987. Actas... San Miguel de Tucumán, Asoc. Geol. Argent. v. II, p. 25-28.

PRECIOZZI, F.; SPOTURNO, J.; HEINZEN, W.; ROSSI, P. 1985. Carta Geológica de la República Oriental del Uruguay. Esc. 1:500.000. 2 ed. Montevideo, Dirección Nacional de Minería y Geología.

RIDING, R. 1991. Classification of microbial carbonates. In: RIDING, R. ed. Calcareous Algae and Stromatolites. Berlin, Springer-Verlag. p. 21-51.

SCOFFIN, T.P. 1987. An Introduction to Carbonate Sediments and Rocks. Glasgow, Blackie. $283 \mathrm{p}$

TUCKER, M.E. 1981. Sedimentary Petrology. An Introduction. Oxford, Blackwell. $260 \mathrm{p}$

TUCKER, M.E. \& WRIGHT, V.P. 1990. Carbonate Sedimentology. Oxford, Blackwell. $450 \mathrm{p}$.

MANUSCRITO A791 Recebido em 13 de setembro de 1993 Revisá 0 do autor em 5 de setembro de 1994 Revisáo aceita em 16 de junho de 1994 


\title{
LA SECUENCIA ARENOSA-PELITICA DE ARROYO DEL SOLDADO
}

\author{
Addenda posterior al trabajo Calizas estromatolíticas y oolíticas y definición de \\ la Formación Arroyo de La Pedrera (? Vendiano, Uruguay)
}

\section{CLAUDIO GAUCHER* Y ALEJANDRO SCHIPILOV**}

Con posterioridad al I Simposio Internacional del Neoproterozoico - Cámbrico de la Cuenca del Plata, se efectuaron nuevos cartografiados geológicos, lo cual permitió ampliar el área abarcada por la Formación Arroyo de la Pedrera.

En la zona del fotoplano Arroyo del Soldado (34 09'S, $5516 \mathrm{~W})$, se encuentra una cadena de cerros con pendiente muy fuerte, alargados y alineados en dirección N15W (por ejemplo, Cerros Gigante, Largo y Espuelitas). La cartografia geológica de la zona y el estudio preliminar de las rocas aflorantes ha mostrado que se trata de potente serie detrítica (la potencia mínima medida es de $700 \mathrm{~m}$, ya que no se observó la base) estrato y granodecreciente. Consta de areniscas silicificadas en la base, pasando concordantemente a arcillitas en el tope. Todo el conjunto ha sufrido compresión con dirección N70-80E, que lo ha plegado en un gran sinclinal de eje subhorizontal N10-20W.

Análisis petrográfico Las areniscas - que constituyen más del $90 \%$ de las rocas aflorantes - han proporcionado la siguiente composición modal: $89 \%$ cuarzo, $6 \%$ yeso, $2 \%$ microclina, $0,5 \%$ ortosa, $1,5 \%$ sericita, $0,5 \%$ plagioclasa y $0,5 \%$ hornblenda, con turmalina y circón como accesorios. Por su parte, la fracción detrítica está compuesta por $90 \%$ cuarzo, $5,5 \%$ microclina + ortosa, $2,5 \%$ sericita, $1 \%$ plagioclasa y $1 \%$ hornblenda, lo que permite clasificarlas como ortocuarcitas. Las areniscas han sufrido fuerte diagénesis, consistente en cementación temprana por yeso y posterior silicificación, lo que le da a la roca gran tenacidad. Se denota también compactación importante, con contactos cóncavo convexos y suturales entre granos, con la consecuente reducción de la porosidad, que es del $10 \%$. La redondez y esfericidad de los clastos, en los casos que se pudo medir, es invariablemente alta.
Estructuras sedimentarias Son abundantes los ripples simétricos - indicativos de la acción del oleaje - y los levemente asimétricos. También se observa estratificación cruzada tipo hummocky y estructuras flaser - linsen en la zona de transición a pelitas.

CONCLUSIONES a. Litológica y estructuralmente el paquete se corresponde con lo definido en esta publicación por Montaña \& Sprechmann para el Miembro Cerros San Francisco de la Formación Arroyo de la Pedrera. Es de destacar que la serie plegada puede seguirse continuamente por casi $100 \mathrm{~km}$, entre Illescas y la zona de Arroyo del Soldado, por lo que se amplía considerablemente el área de afloramiento del citado miembro.

b. Las calizas de Polanco, cuya ubicación se encuentra alineada con los puntos mencionados supra, podría corresponder al Miembro Cerro Victoria de la misma Formación, donde por fenómenos de tectónica en fase rúptil no aflora el miembro silicoclástico. Este hecho confirma la hipótesis formulada en esta publicación acerca de la correlación entre ambas unidades.

c. Ambiente de sedimentación: Es una plataforma somera situada por encima del nivel de las olas del buen tiempo en la base, con disminución de la energía hacia el tope.

d. Paleoclima: Dada la gran madurez textural de las areniscas y la presencia de un $6 \%$ de Yeso, es probable que el paleoclima fuera cálido y por lo tanto la paleolatitud baja.

\footnotetext{
* Facultad de Agronomía, Cátedra de Geología, Avenida. Garzón 780; Montevideo.Uruguay

** Departamento de Paleontología, Facultad de Ciencias, Tristán Narvaja 1674; 11200 Montevideo, Uruguay
} 